\title{
A VARIATIONAL METHOD FOR STARLIKE FUNCTIONS
}

\section{J. A. HUMMEL ${ }^{1}$}

1. One of the most important developments in the study of schlicht functions was the discovery of variational methods. The early work of Marty and the investigations of Schiffer along with those of Schaeffer and Spencer led to the development of powerful machinery for the attack of extremal problems in the class of schlicht functions. Essentially, the Schiffer variational method, when applied to an extremal problem, supplies a differential equation of the form,

$$
\left(\frac{z f^{\prime}(z)}{f(z)}\right)^{2} P\left(\frac{1}{f}\right)=Q(z),
$$

which must be satisfied by the function having the extremal property. A history of the variational method and numerous references may be found in the first chapter of [4].

Let $S$ be the class of all functions $f(z)=z+a_{2} z^{2}+\cdots$ which are regular and schlicht in $|z|<1$, and let $S^{*}$ be the subclass of $S$ consisting of those functions which are starlike (with respect to the origin). The class $S^{*}$ has been studied extensively, but almost all such studies have been based on the fact that for $f(z)$ in $S^{*}$, $\operatorname{Re}\left\{z f^{\prime}(z) / f(z)\right\} \geqq 0$ for all $z$ in the unit circle. The lack of an effective variational method in $S^{*}$ has made much such investigation difficult. Recently Goluzin developed a variational method based on expressing $z f^{\prime}(z) / f(z)$ as a Stieltjes integral [1].

In this paper, a new variational method for starlike functions is described. This method results, as with the Schiffer variation, in a differential equation for the extremal function. However, instead of (1), the equation is of the type

$$
\frac{z f^{\prime}(z)}{f(z)} R(z)=Q(z) .
$$

The right hand sides of (1) and (2) will in general be the same for the same extremal problem.

Presented to the Society, October 26, 1957; received by the editors June 13, 1957 and, in revised form, July $31,1957$.

1 The work reported on here was done while the writer held a National Science Foundation Postdoctoral Fellowship. The writer wishes to express his appreciation to Professor M. Schiffer for many helpful conversations during these investigations. 
The idea behind the variational method is to vary the domain in the image plane so that each boundary point is moved only along a ray through the origin. The resulting domain must then still be starlike, and the new mapping function is still in the class $S^{*}$.

2. The method is based upon the method of interior variation developed by Schiffer [6]. If $w=f(z)$ maps $|z|<1$ onto a simply connected domain $D$ and if $w^{\mathbf{\Lambda}}=w+\rho^{2} \delta w$ maps $C$, the boundary of $D$ onto $C^{\mathbf{\Lambda}}$, the boundary of a new simply connected domain $D^{\mathbf{\Lambda}}$, then the variational formula for the Green's function of $D$ is

$$
\delta g(w, 0)=\operatorname{Re}\left\{\frac{\rho^{2}}{2 \pi i} \oint_{C} p^{\prime}(\eta, w) p^{\prime}(\eta, 0) \delta \eta d \eta\right\}+o\left(\rho^{2}\right),
$$

where $p(\eta, w)$ is the analytic completion of the Green's function of $D$, (see [7]). If $\phi(w)$ is the inverse function of $f(z)$, then $g(w, 0)$ $=-\log |\phi(w)|$. Thus if (3) gives $\delta g(w, 0)=\rho^{2} \operatorname{Re}\{k(w)\}+o\left(\rho^{2}\right)$, where $k(w)$ is analytic in $w$, (3) is easily seen to be equivalent to $\delta \phi(w)=-\rho^{2} \phi(w) k(w)+o\left(\rho^{2}\right)$. Using the fact that $f^{\mathbf{\Lambda}}\left(z^{\mathbf{\Lambda}}\right)=f(z)$, and multiplying by an appropriate constant to preserve the normalization, $f^{\prime}(0)=1$, we have

$$
\delta f(z)=\rho^{2}\left\{z f^{\prime}(z) k[f(z)]-f(z) k(0)\right\}+o\left(\rho^{2}\right) .
$$

The variation in the Schiffer case is $\delta w=e^{i \theta}\left(w-w_{0}\right)^{-1}$, where $w_{0}$ is an interior point of $D$. In our case, to preserve the starlike character of the domain, we make the variation of the form $\delta w=w R[\phi(w)]$, where $R(z)$ is a function which is real and bounded on $|z|=1$. Then on the boundary of $D$, $\delta w$ is purely radial and hence $D^{\mathbf{\Delta}}$ is also starlike. Up to an additive real constant, such a function $R(z)$, having at most one pole in $|z|<1$ at $z=z_{0}$ (a Schottky function), can be written as

$$
R(z)=e^{i \theta} \frac{1-z_{0}^{*} z}{z-z_{0}}+e^{-i \theta} \frac{z-z_{0}}{1-z_{0}^{*} z} .
$$

(Here, and throughout the paper, an asterisk attached to a value indicates the complex conjugate.) Now we have

$$
p(\eta, w)=\log \frac{1-\phi(w)^{*} \phi(\eta)}{\phi(\eta)-\phi(w)},
$$

and

$$
p^{\prime}(\eta, w)=-\frac{\phi^{\prime}(\eta)}{\phi(\eta)-\phi(w)}-\frac{\phi(w)^{*} \phi^{\prime}(\eta)}{1-\phi(w)^{*} \phi(\eta)}
$$


Thus, the integral in (3) becomes

$$
\frac{1}{2 \pi i} \oint_{c}\left[\frac{\phi^{\prime}(\eta)}{\phi(\eta)-\phi(w)}+\frac{\phi(w)^{*} \phi^{\prime}(\eta)}{1-\phi(w)^{*} \phi(\eta)}\right] \frac{\phi^{\prime}(\eta)}{\phi(\eta)} \eta R[\phi(\eta)] d \eta .
$$

The integrand is regular at $\eta=0$ and has singularities only at $\eta=w$ and $\eta=w_{0}\left(\right.$ where $\left.\phi\left(w_{0}\right)=z_{0}\right)$. The residue at $\eta=w$ is

$$
\frac{\phi^{\prime}(w)}{\phi(w)} w R[\phi(w)]
$$

while the residue at $\eta=w_{0}$ is

$$
\left[\frac{\phi^{\prime}\left(w_{0}\right)}{\phi\left(w_{0}\right)-\phi(w)}+\frac{\phi(w)^{*} \phi^{\prime}\left(w_{0}\right)}{1-\phi(w)^{*} \phi\left(w_{0}\right)}\right] \frac{w_{0}}{\phi\left(w_{0}\right)} e^{i \theta}\left[1-z_{0}^{*} \phi\left(w_{0}\right)\right] .
$$

Therefore, with the help of the residue theorem, the analytic function $k(w)$ defined above is

$$
\begin{aligned}
k(w)= & \frac{w \phi^{\prime}(w)}{\phi(w)}\left[e^{i \theta} \frac{1-z_{0}^{*} \phi(w)}{\phi(w)-z_{0}}+e^{-i \theta} \frac{\phi(w)-z_{0}}{1-z_{0}^{*} \phi(w)}\right] \\
& +\left(1-\left|z_{0}\right|^{2}\right)\left\{e^{i \theta} \frac{w_{0} \phi^{\prime}\left(w_{0}\right)}{z_{0}} \frac{1}{\left[z_{0}-\phi(w)\right]}\right. \\
& +e^{-i \theta} \frac{\left.\frac{w_{0}^{*} \phi^{\prime}\left(w_{0}\right)^{*}}{z_{0}^{*}} \frac{\phi(w)}{\left[1-z_{0}^{*} \phi(w)\right]}\right\}+i c,}{}
\end{aligned}
$$

where $w_{0}=f\left(z_{0}\right)$, and $c$ is an arbitrary real constant. However, $c$ remains bounded as $\rho^{2} \rightarrow 0$, since $\delta \phi(w) \rightarrow 0$ as $\rho^{2} \rightarrow 0$. Therefore, from (4), except for a term, $\rho^{2} i c\left\{z f^{\prime}(z)-f(z)\right\}$, we have

$$
\begin{aligned}
\delta f(z)= & \rho^{2}\left(1-\left|z_{0}\right|^{2}\right)\left\{e^{i \theta} \frac{z f(z)}{z_{0}\left(z-z_{0}\right)}+e^{-i \theta} \frac{z f(z)}{\left(1-z_{0}^{*} z\right)}\right. \\
& -e^{i \theta}\left(\frac{f\left(z_{0}\right)}{z_{0} f^{\prime}\left(z_{0}\right)}\right)\left[\frac{z f^{\prime}(z)}{z-z_{0}}+\frac{f(z)}{z_{0}}\right] \\
& \left.+e^{-i \theta}\left(\frac{f\left(z_{0}\right)}{z_{0} f^{\prime}\left(z_{0}\right)}\right)^{*} \frac{z^{2} f^{\prime}(z)}{\left(1-z_{0}^{*} z\right)}\right\}+o\left(\rho^{2}\right) .
\end{aligned}
$$

The missing term may be dropped since it can be accounted for by an allowable variation of the form $e^{-i \epsilon} f\left(e^{i \epsilon} z\right)$, giving $\delta f=i \epsilon\left[z f^{\prime}(z)-f(z)\right]$ $+o(\epsilon)$. Thus, (6) is the required variational formula.

3. Formula (6) can now be used to prove

THEOREM 1. Let $F\left(a_{2}, a_{3}, \cdots, a_{n}\right)$ be any function of the $n-1$ vari- 
ables $a_{2}, a_{3}, \cdots, a_{n}$ having a continuous derivative in each variable. Then any $f(z)=z+a_{2} z^{2}+a_{3} z^{3}+\cdots \quad$ in $S^{*}$ which maximizes $\operatorname{Re}\left\{F\left(a_{2}, \cdots, a_{n}\right)\right\}$ must be of the form

$$
f(z)=z /\left(\prod_{\nu=1}^{m}\left(1-\kappa_{\nu} z\right)^{\mu_{\nu}}\right)
$$

where $\left|\kappa_{\nu}\right|=1, \mu_{\nu} \geqq 0$, for all $\nu, \sum_{\nu=1}^{m} \mu_{\nu}=2$, and $m \leqq n-1$.

Proof. The compactness of $S^{*}$ shows that there is a function in $S^{*}$ which maximizes $\operatorname{Re}\left\{F\left(a_{2}, \cdots, a_{n}\right)\right\}$. If we let $\lambda_{\nu}=\partial F / \partial a_{\nu}$ at the point $\left(a_{2}, \cdots, a_{n}\right)$ determined by this function, it is clear that the problem is equivalent to maximizing $\operatorname{Re}\left\{\sum_{\nu=2}^{n} \lambda_{\nu} a_{\nu}\right\}$ in $S^{*}$. From (6)

$$
\begin{aligned}
\delta a_{n}= & \rho^{2}\left(1-\left|z_{0}\right|^{2}\right)\left\{-e^{i \theta} \frac{1}{z_{0}} \sum_{\mu=1}^{n-1} \frac{a_{\mu}}{z_{0}^{n-\mu}}+e^{-i \theta} \frac{1}{z_{0}^{*}} \sum_{\mu=1}^{n-1} a_{\mu} z_{0}^{* n-\mu}\right. \\
& +e^{i \theta}\left(\frac{f\left(z_{0}\right)}{z_{0} f^{\prime}\left(z_{0}\right)}\right)\left[\frac{1}{z_{0}} \sum_{\mu=1}^{n-1} \frac{\mu a_{\mu}}{z_{0}^{n-\mu}}+\frac{(n-1) a_{n}}{z_{0}}\right] \\
& \left.+e^{-i \theta}\left(\frac{f\left(z_{0}\right)}{z_{0} f^{\prime}\left(z_{0}\right)}\right)^{*} \frac{1}{z_{0}^{*}} \sum_{\mu=1}^{n-1} \mu a_{\mu} z_{0}^{* n-\mu}\right\}+o\left(\rho^{2}\right) .
\end{aligned}
$$

For the extremal $f(z)$ we must have $\operatorname{Re}\left\{\sum_{\nu=2}^{n} \lambda_{\nu} \delta a_{\nu}\right\} \leqq 0$ for each $\theta$. Since we can take the complex conjugate of each term inside the brackets having a factor $e^{-i \theta}$ without changing the real part, the extremal function must satisfy the differential equation

$$
\frac{z f^{\prime}(z)}{f(z)} R(z)=Q(z),
$$

where

$$
\begin{aligned}
& R(z)=\sum_{\nu=2}^{n}\left[\lambda_{\nu} \sum_{\mu=1}^{\nu-1} \frac{a_{\mu}}{z^{\nu-\mu}}-\lambda_{\nu}^{*} \sum_{\mu=1}^{\nu-1} a_{\mu}{ }^{*} z^{\nu-\mu}\right], \\
& Q(z)=\sum_{\nu=2}^{n}\left[\lambda_{\nu} \sum_{\mu=1}^{\nu-1} \frac{\mu a_{\mu}}{z^{\nu-\mu}}+(\nu-1) \lambda_{\nu} a_{\nu}+\lambda_{\nu}^{*} \sum_{\mu=1}^{\nu-1} \mu a_{\mu}^{*} z^{\nu-\mu}\right] .
\end{aligned}
$$

The usual argument with the variation $e^{-i \theta} f\left(e^{i \theta} z\right)$ shows that $\sum_{\nu=2}^{n}(\nu-1) \lambda_{\nu} a_{\nu}$ is real (see $[5$, p. 498]). Hence on $|z|=1, Q(z)$ is real, $R(z)$ is imaginary, and thus $z f^{\prime} / f$ is pure imaginary. Since $z f^{\prime} / f$ $=-i \partial \log f\left(r e^{i \theta}\right) / \partial \theta$, this means that the boundary of $D$ consists only of radial segments, and in particular, $D$ thus has no exterior.

Also from (9), $z f^{\prime} / f$ is a rational function. Since it is regular in $|z|<1$, by the reflection principal it has poles only on $|z|=1$. Therefore we can write 


$$
\frac{f^{\prime}(z)}{f(z)}=\frac{1}{z}+\sum_{\nu=1}^{m} \frac{\mu_{\nu} \kappa_{\nu}}{1-\kappa_{\nu} z},
$$

$$
m \leqq 2 n-2,\left|\kappa_{\nu}\right|=1
$$

Integrating, $f(z)$ must be of the form (7). Now $z f^{\prime} / f=1$ at $z=0$, and hence must be -1 at $z=\infty$. Letting $z \rightarrow \infty$ in (11), we have $\sum \mu_{\nu}=2$. Also, $\kappa_{\nu} z\left(1-\kappa_{\nu} z\right)^{-1}$ has real part $-1 / 2$ on $|z|=1$, and its imaginary part becomes infinite as $z \rightarrow \kappa_{\nu}^{*}$. Since $z f^{\prime} / f$ is imaginary on $|z|=1$, letting $z \rightarrow \kappa_{\nu}^{*}$, each $\mu_{\nu}$ must be real. The fact that $\mu_{\nu} \geqq 0$ follows from (7) since $f(z)$ cannot tend to zero as $z \rightarrow K_{\nu}^{*}$.

Now $z f^{\prime} / f$ has $m$ simple zeros on $|z|=1$ corresponding to the tips of the $m$ slits. From (9), $Q(z)$ must have zeros at these points. Also $z f^{\prime} / f$ has $m$ simple poles at $\kappa_{1}^{*}, \cdots, \kappa_{m}^{*}$, corresponding to the ends of the slits at $\infty . R(z)$ must have zeros at these points. There remains $2 n-2-m$ zeros which $R(z)$ and $Q(z)$ have in common.

We next show that $Q(z)$ is positive on $|z|=1$ in a deleted neighborhood of each of the points $\kappa_{\nu}^{*}$ by means of a Julia variation [2]. Let $z_{0}$ be the closest zero of $Q(z)$ on one side of $\kappa_{\nu}^{*}$, corresponding to $w_{0}$ on one side of the $\nu$ th slit. Make the Julia variation $\delta w= \pm \epsilon i\left(w-w_{0}\right)$ on that side of the slit from $w_{0}$ to $\infty$. The sign is chosen so that this portion of the slit is opened out into a wedge. This preserves the starlike character of $D$. Then it can be shown that

$$
\delta \operatorname{Re}\left\{\sum_{\nu=2}^{n} \lambda_{\nu} a_{\nu}\right\}=\operatorname{Re}\left\{\oint_{C} Q(z)\left[\frac{1}{2 \pi i} \frac{\delta w d w}{f^{\prime}(z)^{2} z^{2}}\right]\right\},
$$

see [5, Equation (2.18)]. For the variation used here, this integral converges, and the expression in the square brackets is always negative. Hence $Q(z)$ must be positive for the extremal function.

If we go from $\kappa_{\nu}^{*}$ to $\kappa_{\nu+1}^{*}, Q(z)$ is positive at the two end points, and real in between. Hence it has an even number of zeros in this interval. But it has one corresponding to the end point of the slit, and therefore it has, in all, at least $2 m$ zeros on $|z|=1$. But $Q(z)$ has only $2 n-2$ zeros altogether. Hence $m \leqq n-1$. This completes the proof of the theorem.

This theorem could also be proved by an appeal to the PickNevanlinna interpolation problem. In this regard, the paper of Nehari and Netanyahu [3] may be consulted.

4. The differential equation (9) can be used to find the coefficient regions of the starlike functions. The writer is preparing another paper in which this is done. It turns out that for fixed $a_{2}, a_{3}, \cdots$, $a_{n-1}$, the coefficient $a_{n}$ lies in a circle. In particular, if $\left|a_{2}\right| \leqq 2$ and if $f(z)=z+a_{2} z^{2}+a_{3} z^{3}+\cdots$ is in $S^{*}$, then $a_{3}$ is contained in the closed circle 


$$
a_{3}=3 a_{2}^{2} / 4+\left(4-\left|a_{2}\right|^{2}\right) r e^{i \theta} / 4, \quad 0 \leqq r \leqq 1,0 \leqq \theta \leqq 2 \pi .
$$

This result may be compared with that of Schaeffer and Spencer for general schilicht functions [4].

These results can also be applied to the study of convex functions, since a normalized function $f(z)$ is convex if and only if $z f^{\prime}(z)$ is starlike. For example, the result in (12) shows that for any starlike function $\left|4 a_{3}-3 a_{2}^{2}\right| \leqq 4$, and hence we have a proof of a conjecture of V. Singh that for any convex function $\left|a_{3}-a_{2}^{2}\right| \leqq 1 / 3$.

The variational formula (6) may be applied to many other problems. However, at this writing the author has only a limited number of ways of getting information out of the resulting differential equation. As further work is done, improved results should be obtained.

Finally it may be remarked that the device used here to obtain a starlike variation should be adaptable to the study of functions schlicht and starlike in an annulus.

\section{REFERENCES}

1. G. M. Goluzin, On a variational method in the theory of analytic functions, Leningrad. Gos. Univ. Uč. Zap. vol. 144 Ser. Mat. Nauk 23 (1952) pp. 85-101 (Russian).

2. G. Julia, Sur une équation aux dérivées fonctionnelles liée à la représentation conforme, Ann. Êcole Norm. Sup. (3), vol. 39 (1922) pp. 1-28.

3. Z. Nehari and E. Netanyahu, On the coefficients of meromorphic schlicht functions, Proc. Amer. Math. Soc. vol. 8 (1957) pp. 15-23.

4. A. C. Schaeffer and D. C. Spencer, Coefficient regions for schlicht functions, Amer. Math. Soc. Colloquium Publications, vol. 35, 1950.

5. A. C. Schaeffer, M. Schiffer, and D. C. Spencer, The coefficient regions of schlicht functions, Duke Math. J. vol. 16 (1949) pp. 493-527.

6. M. Schiffer, A method of variation within the family of simple functions, Proc. London Math. Soc. vol. 44 (1938) pp. 432-449.

7. - Applications of variational methods in the theory of conformal mapping, Proceedings of the Symposia in Applied Mathematics, 1956 (to appear).

UNIVERSITY OF MARYLAND 